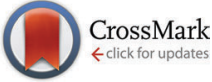

Cite this: Phys. Chem. Chem. Phys., $2015,17,32375$

Received 31st August 2015, Accepted 29th October 2015 DOI: $10.1039 / c 5 c p 05196 \mathrm{~g}$

www.rsc.org/pccp

\title{
Investigation of the fragmentation of core-ionised deoxyribose: a study as a function of the tautomeric form
}

\author{
Marie-Anne Hervé du Penhoat, ${ }^{\star a}$ Krishna Kamol Ghose, ${ }^{b}$ Marie-Pierre Gaigeot, ${ }^{\text {bc }}$ \\ Rodolphe Vuilleumier, ${ }^{d}$ Kentaro Fujii, ${ }^{\mathrm{e}}$ Akinari Yokoya ${ }^{\mathrm{e}}$ and Marie-Françoise Politis ${ }^{\mathrm{b}}$
}

\begin{abstract}
We have investigated the gas phase fragmentation dynamics following the core ionisation of 2-deoxy-Dribose (dR), a major component in the DNA chain. To that aim, we use state-of-the-art ab initio Density Functional Theory-based Molecular Dynamics simulations. The ultrafast dissociation dynamics of the core-ionised biomolecule, prior Auger decay, is first modelled for 10 fs to generate initial configurations (atomic positions and velocities) for the subsequent dynamics of the doubly ionised biomolecule in the ground state. The furanose, linear and pyranose conformations of $\mathrm{dR}$ were investigated. We show that fragmentation is relatively independent of the atom struck or of the duration of the core vacancy, but depends rather critically on the molecular orbital removed following Auger decay.
\end{abstract}

\section{Introduction}

Ionising radiations (electromagnetic radiation, beams of electrons, protons, or heavy ions) are commonly used in cancer therapy to irradiate "risk zones" or tumours. It is well understood that energy deposition by ionising radiation at various DNA sites, such as sugars and bases, can lead to permanent molecular changes, and some of those are known to be the initiating events for the cells inactivation or the formation of chromosomal aberrations. Establishing the origins of radiation induced DNA damage thus seems an important step for the application of these therapies and for the investigation of their possible undesirable side effects.

The purpose of this paper is to understand, at the atomic level, the direct effects of ionising radiations on DNA building blocks. Here, we will focus on describing, by means of quantumclassical theoretical methods, the fragmentation processes of core ionised deoxyribose (dR), at the molecular scale, since the induction of core holes in the DNA molecule was shown to lead to major lethal effects in cells. ${ }^{1-6}$ The overall proposed mechanism is the following: core hole states have a strongly dissociative character. They may induce ultra-fast dissociation reactions in which the nuclear

\footnotetext{
${ }^{a}$ IMPMC, Sorbonne Universités - UPMC Univ Paris 06, UMR CNRS 7590, Muséum National d'Histoire Naturelle, IRD UMR 206, 4 Place Jussieu, 75005 Paris, France. E-mail: penhoat@impmc.upmc.fr; Fax: +33 1442737 85; Tel: +33144277502

${ }^{b}$ LAMBE, UMR CNRS 8587, Université d'Evry val d'Essonne, 91025 Evry, France

${ }^{c}$ Institut Universitaire de France IUF, 103 Blvd St Michel, 75005 Paris, France

${ }^{d}$ Ecole Normale Supérieure, Département de Chimie, UMR 8640 CNRS-ENS-UPMC, 75005 Paris, France

${ }^{e}$ Advance Science Research Center, Japan Atomic Energy Agency, Tokai, Japan
}

dynamics takes place on time scales comparable to the lifetime of the core vacancies, which is about 8 and 5 fs in the case of carbon and oxygen atoms, respectively. ${ }^{7}$ Moreover, in the relaxation process following the inner-shell ionisation events, Auger transition probabilities are nearly $100 \%$ in DNA constituent atoms, leading to the multi-ionisation of the atom struck and to the emission of two low energy electrons, the photo- and Auger electrons, which can in turn, locally damage DNA.

Many experiments have been performed in the gas phase in order to understand the mechanisms of fragmentation of sugars after K-shell ionisation and/or multiple ionisation events (by ionisation or charge transfer). ${ }^{8-10}$ Several authors have pointed out that the dissociation process of a molecule after K-shell excitation or ionisation in light atoms does not seem to depend on the position nor on the nature of the core-ionised atom but rather on the molecular orbitals (MO) ionised after Auger decay. ${ }^{10-13}$ In order to understand this experimental fact, we have simulated core holes dynamics followed, after Auger decay, by double hole dynamics.

Different theoretical approaches have been developed in order to describe the fragmentation processes of doubly ionised biomolecules. In previous studies, we have used Time-Dependent Density Functional Theory (TD-DFT) molecular dynamics (MD) to remove electrons from selected MO and follow in time the nuclear and electrons dynamics. ${ }^{14-16}$ This method is computationally demanding but provides the physical origin of bond breakage. Born Oppenheimer and Car Parrinello MD of ground state doublyionised isolated uracil were also performed at high temperature in order to take into account the energy deposit by the incoming high velocity ion. ${ }^{15}$ Other methods were recently developed. 
<smiles>C=CC1C[C@@H](O)[C@@H](O)CO1</smiles>

Deoxyribopyranose
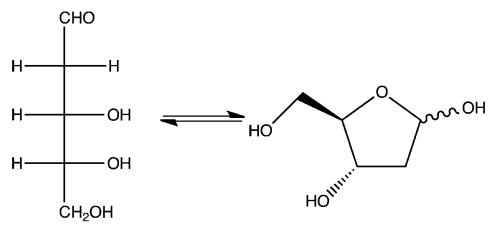

Deoxyribofuranose
Fig. 1 Structures of the pyranose, linear and furanose forms of 2-deoxyD-ribose.

One of them ${ }^{17}$ is a simple statistical approach based on the enumeration of assumed equiprobable pathways of breakage of doubly ionised molecules. The other one ${ }^{18}$ is based, like ours, on molecular dynamics simulations. Initial states are generated with internal energy, i.e. temperature, corresponding to a sampling of energies within a range of estimated energy deposits in the molecule after core ionisation and different Auger decay channels. Starting from these different initial configurations (atomic positions and velocities), ground state double hole MD are performed. These methods provide the different fragmentation pathways but do not describe the driving mechanisms (electron dynamics).

As pointed out in experimental and theoretical works, ${ }^{10}$ the 2-deoxy-D-ribose molecule may be found under different conformations with regards to the environment (Fig. 1). In the DNA backbone, this monosaccharide is mainly in the furanose form constructed around a 5-membered ring with $\mathrm{CH}_{2} \mathrm{OH}$ and $\mathrm{OH}$ substituents. Such a conformation has been considered in our previous work ${ }^{19}$ and is worth investigating with regards to the behaviour of DNA building blocks. However, in gas-phase experiments after being heated in an oven, the pyranose form appears dominant ${ }^{20}$ and comparison with experimental data should take this conformation into account. The 2-deoxy-Dribose may also exist under a linear form. To understand the impact of the sugars conformation on their dissociation, we have investigated in parallel the fragmentation induced by K-shell ionisation followed by Auger processes for the furanose, pyranose and linear conformations of 2-deoxy-D-ribose.

\section{Material and methods}

We explicitly model core-ionisation of the carbon and oxygen atoms of the sugar as initial electronic conditions, and follow the subsequent dynamics of the ionised sugar by Born-Oppenheimer Molecular Dynamics simulations (BOMD). These simulations were performed with the plane wave Kohn Sham based DFT code CPMD ${ }^{21}$ Core electrons were replaced by pseudopotentials of the Trouiller-Martins form. ${ }^{22}$ The Kleinman-Bylander integration scheme was used for all atom type, and the plane-wave basis set was truncated at an energy cutoff of 90 Rydberg (Ry: 1 Ry $=2.17989 \times 10^{18} \mathrm{~J}$ ). The exchange and correlation energy was calculated using the generalized gradient approximation (GGA) functional BLYP. ${ }^{23,24}$ The long-range electrostatic potential was computed using the standard Ewald summation scheme and a compensating background was thus used for the

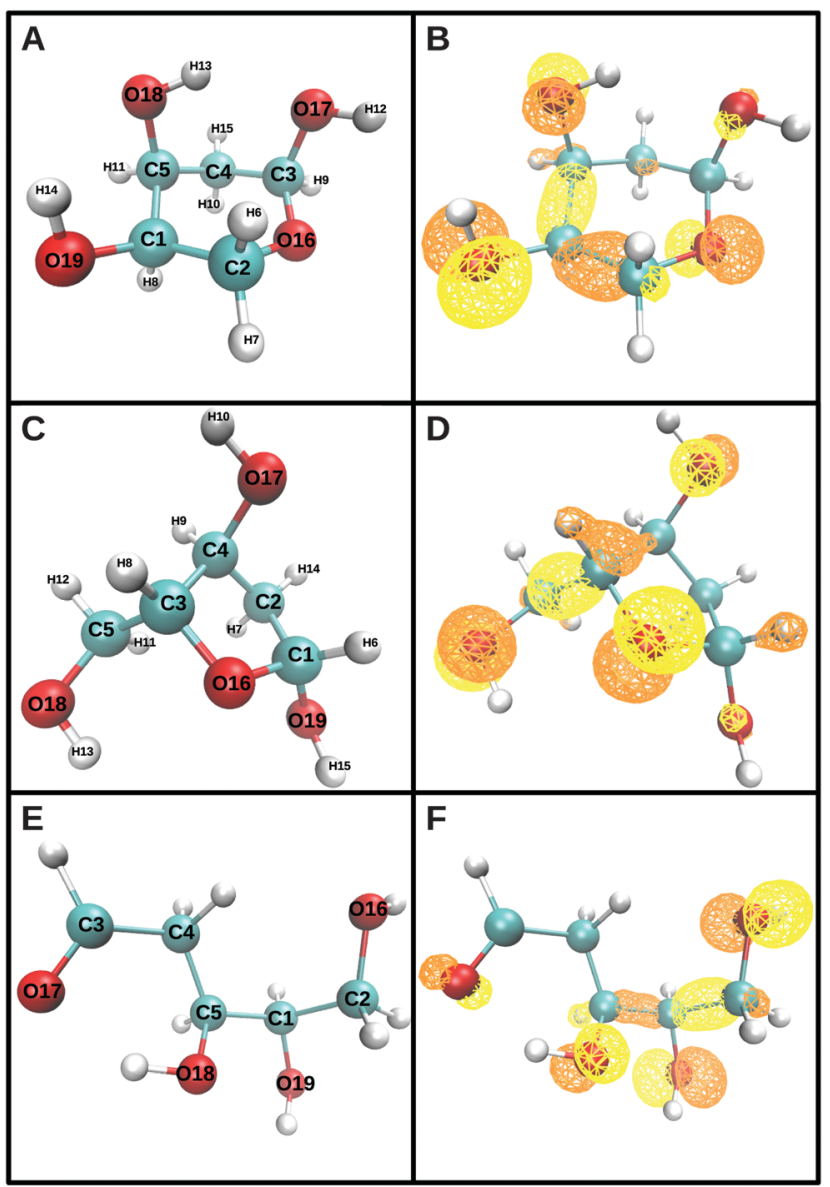

Fig. 2 The three deoxyribose conformations investigated: A and B, pyranose; $C$ and $D$, furanose; and $E$ and $F$, linear. $A, C$ and $E$, atom labelling; and $B, D$ and $\mathrm{F}, \mathrm{HOMO}$ negative (yellow) and positive (orange) contours. $\mathrm{C}$ blue, $\mathrm{O}$ red, $\mathrm{H}$ white.

charged simulations. The analysis of the fragments charges were performed in the Bader scheme. ${ }^{25}$

The three different conformations of deoxyribose investigated in this study are presented in Fig. 2. All simulations presented here model the three isomeric forms of 2-deoxy-D-ribose in a cubic box of size $L=20 \AA$. The initial atomic configurations (positions and velocities) were generated at the end of a $10 \mathrm{ps}$ equilibration run with the Car-Parrinello MD algorithm thermostated at $320 \mathrm{~K}$ by a Nosé-Hoover chain. For the pyranose and furanose forms, ten different initial configurations were selected at 484 fs intervals along a neutral BOMD, taking time steps of $0.12 \mathrm{fs}$. For the linear form, two different initial configurations were selected at $1 \mathrm{ps}$ intervals along a neutral BOMD, taking time steps of $0.12 \mathrm{fs}$.

The dissociation induced by the ionisation of core electrons of dR atoms was modelled in two steps: (1) the ultrafast dissociation reactions induced by the core vacancy, before Auger decay, was simulated first. This step was described by replacing the $1 \mathrm{~s}^{2}$ pseudopotential of one atom of the target molecule with a pseudopotential for a $1 \mathrm{~s}^{1}$ core-hole state, as detailed in ref. 26 . A global charge equal to unity was added to the system to take into account the missing electron. BOMD simulations of the 
core-ionised system were then performed for $9.6 \mathrm{fs}$, taking time steps of 0.024 fs. One initial configuration was selected for each $\mathrm{dR}$ conformation. Core-holes were placed successively on each of the five carbon and four oxygen atoms of the $\mathrm{dR}$ molecule. Consequently, nine core-hole trajectories were generated for each dR conformation. (2) The dissociation dynamics of the doubly-ionized dR molecule, after Auger decay, was then modelled. In the second step, we thus started from atomic configurations generated during the first step (at $t=4.8 \mathrm{fs}$ and $t=9.6 \mathrm{fs}$ ) but changing the charge of the system from +1 (step 1) to +2 (step 2, this step). BOMD of ground state $\mathrm{dR}^{2+}$ were performed for several hundreds of femtoseconds, taking time steps of 0.024 fs. Two different $\mathrm{dR}^{2+}$ trajectories were thus generated for each core-hole trajectory, replacing the core hole by a double vacancy at either $t=4.8$ fs or $t=9.6$ fs.

In parallel, we have investigated the fragmentation of doubly-ionized dR (without prior core ionisation) starting from several different initial configurations and removing the two electrons from the highest occupied MO (HOMO, see Fig. 2). BOMD were performed for several hundreds of femtoseconds, taking time steps of 0.024 fs. These dynamics will provide a benchmark to compare to the fragmentation induced by double ionisation events resulting from core ionisation and subsequent Auger decay.

\section{Results and discussion}

\subsection{Pyranose conformation}

3.1.1 Double hole dynamics. BOMD of ground state $\mathrm{dR}^{2+}$ in the pyranose conformation were performed for at least $300 \mathrm{fs}$, starting from ten different initial configurations. Two different fragmentation patterns were found.

The most probable pathway, which corresponds to eight out of ten cases, is illustrated in Fig. 3. In this particular dynamics, the $\mathrm{C} 1 \mathrm{C} 2$ and $\mathrm{C} 3 \mathrm{O} 16$ bonds first dissociate at $\mathbf{1 5 . 3}$ and $\mathbf{5 2 . 5} \mathrm{fs}$, respectively. In the following, $\mathrm{CO}$ and $\mathrm{CC}$ bonds will be considered broken when the bond distance exceeds $2 \AA$. A criteria of $1.5 \AA$ is used for $\mathrm{OH}$ bonds. ${ }^{27} \mathrm{~A} \mathrm{CH}_{2} \mathrm{O}$ fragment (30 amu) is thus formed, which is almost neutral (charge about $+0.3 e$ ). The remaining $\mathrm{C}_{4} \mathrm{H}_{8} \mathrm{O}_{3}{ }^{1.7+}$ fragment is shown to dissociate after 408 fs to form
$\mathrm{C}_{2} \mathrm{H}_{4} \mathrm{O}_{2}{ }^{0.9+}(60 \mathrm{amu})$ and $\mathrm{C}_{2} \mathrm{H}_{4} \mathrm{O}^{0.8+}(44 \mathrm{amu})$ fragments. In all eight cases, the fragmentation of the $\mathrm{C} 1 \mathrm{C} 2$ bond is the fastest, between 15.3 to $17.9 \mathrm{fs}$, while the C3O16 bond breaks after 46 to $168 \mathrm{fs}$. The second fragmentation step takes place at much longer times and was only observed in two cases, at 408 and 474 fs. The $\mathrm{CH}_{2} \mathrm{O}^{0.3+}$ fragment sometimes exited the simulation box. When it was more than $12 \AA$ away from $\mathrm{C}_{4} \mathrm{H}_{8} \mathrm{O}_{3}{ }^{1.7+}$, typically after about $350-410 \mathrm{fs}$, the $\mathrm{C}_{4} \mathrm{H}_{8} \mathrm{O}_{3}$ fragment was isolated and BOMD were performed for the doubly ionized system.

The second fragmentation pathway is shown in Fig. 4. In this particular dynamics, the C1C5 bond dissociates at $19.2 \mathrm{fs}$. This bond was found to dissociate at $18.2 \mathrm{fs}$, in the other case leading to the same fragmentation pattern. As a consequence, the $\mathrm{dR}^{2+}$ molecule becomes linear and this configuration remains stable within the few picoseconds investigated in this paper.

In conclusion, two different fragmentation pathways are found, initiated by the rupture of either the $\mathrm{C} 1 \mathrm{C} 2$ or the $\mathrm{C} 1 \mathrm{C} 5$ bond. The HOMO orbital is localized on these two bonds at the beginning of the dynamics (Fig. 2B) and there appears to be a competition in the fragmentation induced in these two bonds by the removal of the HOMO.

3.1.2 Core hole dynamics. Fragmentation was never observed to occur within the lifetime of inner shell vacancies in the case of isolated deoxyribopyranose. The bonds involving the core-ionised atom usually exhibit the largest length variations. In the case of carbon-K vacancies (labelled $\mathrm{C}^{*}$ ), $\mathrm{C}^{*} \mathrm{H}$ bond distances typically decrease from $1.1 \AA$ to $1.0 \AA$ at $t=4.8 \mathrm{fs}$, and increase again up to $1.1 \AA$ at 9.6 fs. $\mathrm{C}^{*} \mathrm{O}$ bond decrease slightly by 0.01 to $0.06 \AA$ A during the 9.6 fs dynamics, whereas $\mathrm{C}^{*} \mathrm{C}$ bond distance variations range from -0.03 to $0.09 \AA$ at $t=9.6 \mathrm{fs}$. The largest variations are for $\mathrm{C} 1 \mathrm{C} 5$ when a core hole is placed on either $\mathrm{C} 1$ or $\mathrm{C} 5$ and $\mathrm{C} 3 \mathrm{C} 4$ when a core hole is placed on $\mathrm{C} 4$.

In the case of oxygen- $\mathrm{K}$ vacancies (labelled $\mathrm{O}^{*}$ ), $\mathrm{O}^{*} \mathrm{H}$ bonds remain fairly constant. In contrast, $\mathrm{CO}^{*}$ bonds exhibit relatively large increase by 0.08 to $0.33 \AA$ as shown in Table 1 . The smallest variations correspond to the localization of the core hole on O16, which is located within the ring, while the largest corresponds to a core hole on O17.

3.1.3 Double hole dynamics following core ionisation. We propagated the core-hole states for $9.6 \mathrm{fs}$, probing the different carbon and oxygen atoms in the dR molecule. We then switched

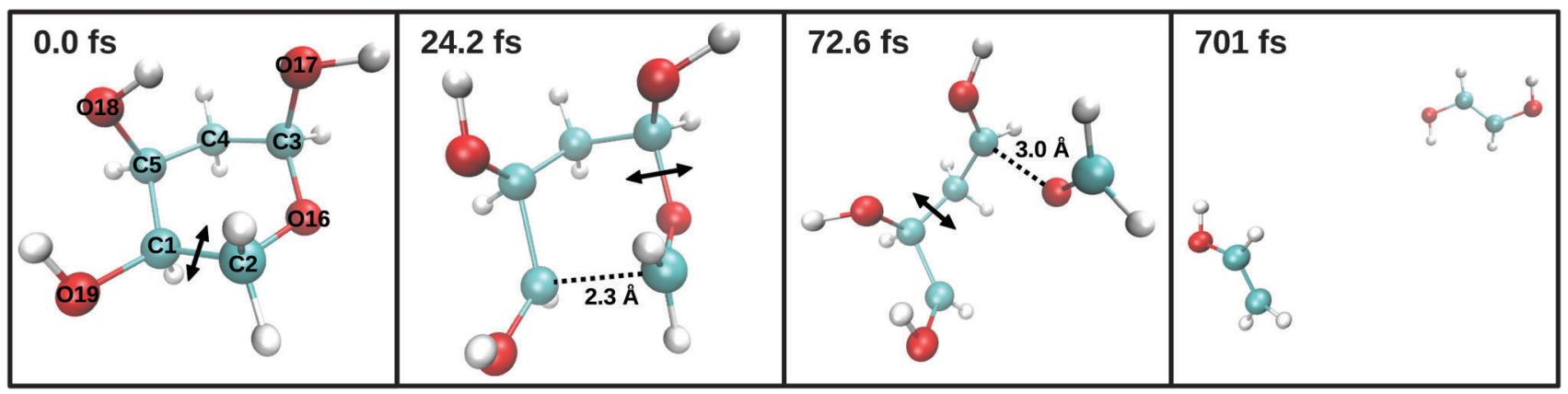

Fig. 3 BOMD trajectory initiated from an electronic configuration where two electrons were removed from the HOMO of the deoxyribopyranose molecule (left, $t=0$ ). The $\mathrm{C}_{4} \mathrm{O}_{3} \mathrm{H}_{8}{ }^{2+}$ fragment was isolated during the last $290 \mathrm{fs}$ of the dynamics. Bond breakage positions are marked by arrows. $\mathrm{C}$ blue, $\mathrm{O}$ red, $\mathrm{H}$ white. 


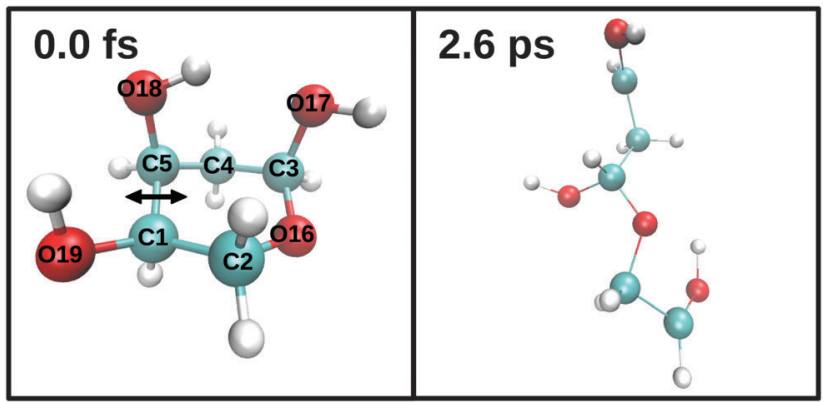

Fig. 4 BOMD trajectory initiated from an electronic configuration where two electrons were removed from the HOMO of the deoxyribopyranose molecule. $\mathrm{C}$ blue, $\mathrm{O}$ red, $\mathrm{H}$ white.

Table 1 Pyranose conformation. Oxygen core-hole dynamics

\begin{tabular}{lllll}
\hline \multirow{2}{*}{ Atom } & & \multicolumn{3}{l}{ Bond distance $\AA$} \\
\cline { 3 - 5 } & Bond & $t=0$ & $t=4.8 \mathrm{fs}$ & $t=9.6 \mathrm{fs}$ \\
\hline O16 & C2-O16* & 1.47 & 1.51 & 1.59 \\
& C3-O16* & 1.44 & 1.48 & 1.61 \\
\multirow{2}{*}{ O17 } & C3-O17 & 1.46 & 1.42 & 1.35 \\
& C3-O17* & 1.46 & 1.55 & 1.79 \\
& C2-O16 & 1.47 & 1.49 & 1.54 \\
O18 & C3-O16 & 1.44 & 1.38 & 1.26 \\
O19 & C5-O18* & 1.46 & 1.52 & 1.68 \\
& C1-O19* & 1.44 & 1.50 & 1.66 \\
\hline
\end{tabular}

to doubly-charged state after either 4.8 or 9.6 fs of core-hole states. The initial configuration is the same as in Fig. 4, which led to the linearisation of the doubly ionised molecule, without prior core ionisation.

When the core hole is located on C1, C4 or C5 during either 4.8 fs or 9.6 fs, or on C3 and 017 during 4.8 fs before double ionisation, the $\mathrm{dR}^{2+}$ molecule breaks at the C1C5 bond and remains linear. The dissociation of $\mathrm{dR}^{2+}$ is thus not modified by the slight changes in the molecules geometry induced by the presence of the core vacancy.

When the core hole is located on C2, O16, O18 or O19 during either 4.8 or $9.6 \mathrm{fs}$, or on C3 during 9.6 fs before double ionisation, the $\mathrm{dR}^{2+}$ molecule breaks at the $\mathrm{C} 1 \mathrm{C} 2$ and $\mathrm{C} 3 \mathrm{O} 16$ bonds, leading to the formation of $\mathrm{CH}_{2} \mathrm{O}^{0.3+}(30 \mathrm{amu})$ and $\mathrm{C}_{4} \mathrm{H}_{8} \mathrm{O}_{3}{ }^{1.7+}$ (104 amu) fragments. In the case of core holes on $\mathrm{O} 16$ (4.8 fs), $\mathrm{O} 18$ (9.6 fs) and $\mathrm{O} 19$ (4.8 and $9.6 \mathrm{fs}$ ), the remaining linear fragment further dissociates after about 400 fs to form $\mathrm{C}_{2} \mathrm{H}_{4} \mathrm{O}^{0.8+}(44 \mathrm{amu})$ and $\mathrm{C}_{2} \mathrm{H}_{4} \mathrm{O}_{2}{ }^{0.9+}(60 \mathrm{amu})$ fragments. These fragmentation patterns were also observed in $\mathrm{dR}^{2+}$, without prior core ionisation, but starting from other initial configurations. It thus appears, that the molecules geometry modifications induced by the presence of these core vacancies favour the second identified dissociation pathway for $\mathrm{dR}^{2+}$ in its ground state.

Finally, when a core hole is placed on 017 during $9.6 \mathrm{fs}$, the fragmentation pattern is completely different, as shown in Fig. 5. First, the $\mathrm{C} 3 \mathrm{O} 17$ bond dissociates at $t=13.7$ fs to yield an OH fragment, then the C1C5 bond dissociates ( $t=29.1 \mathrm{fs})$. This is followed by the dissociation of the $\mathrm{O} 18 \mathrm{H} 13$ bond at $t=57.4$ fs. The $\mathrm{H} 13$ atom reacts with the $\mathrm{OH}$ fragment to form an $\mathrm{H}_{2} \mathrm{O}$ molecule. Finally the C5C4 bond breaks at $t=241 \mathrm{fs}$, releasing a $\mathrm{CHO}$ fragment. This whole set of events leads to the formation of 3 fragments: $\mathrm{H}_{2} \mathrm{O}^{0.2+}(18 \mathrm{amu}), \mathrm{CHO}^{0.8+}(29 \mathrm{amu})$ and $\mathrm{C}_{4} \mathrm{H}_{7} \mathrm{O}_{2}{ }^{+}(87 \mathrm{amu})$. The presence of a core vacancy on $\mathrm{O} 17$ during $9.6 \mathrm{fs}$ induced an increase of the $\mathrm{C} \mathrm{O} 17^{*}$ bond by about $0.3 \AA$ (see Table 1), which could be at the origin of this new fragmentation pathway for the subsequent $\mathrm{dR}^{2+}$ dynamics. This case corresponds to the largest $\mathrm{CO}^{*}$ bond increase observed during the core hole state for deoxyribopyranose.

As a conclusion, in all cases but one, the fragmentation pathways are the same as those found for ground state $\mathrm{dR}^{2+}$, without prior core ionisation.

\subsection{Furanose conformation}

3.2.1 Double hole dynamics. BOMD of the ground state $\mathrm{dR}^{2+}$ were performed for $380 \mathrm{fs}$, starting from ten different initial configurations. The fragmentation pattern is similar in all cases. As illustrated in Fig. 6, the C5C3 bond breaks between 14.6 and 38.2 fs leading to the release of a $\mathrm{CH}_{3} \mathrm{O}^{+}$fragment (mass $31 \mathrm{amu}$ ). In a second step, the ring opens up at the C1O16 bond after 27.1 to 59.5 fs (depending on the trajectory). Large C2C4 bond vibration are observed (i.e. resulting in bond distances larger than $2 \AA$ in some cases).

3.2.2 Core hole dynamics. Fragmentation was never observed within the lifetime of inner shell vacancies in isolated deoxyribopfuranose. In the case of carbon core-holes $\left(\mathrm{C}^{*}\right)$, the core vacancy induces only very slight modifications of the $\mathrm{dR}$ geometry. The largest variations are observed for $\mathrm{C}^{*} \mathrm{H}$ bonds. These bond distances decrease between 0.09 and $0.17 \AA$ during

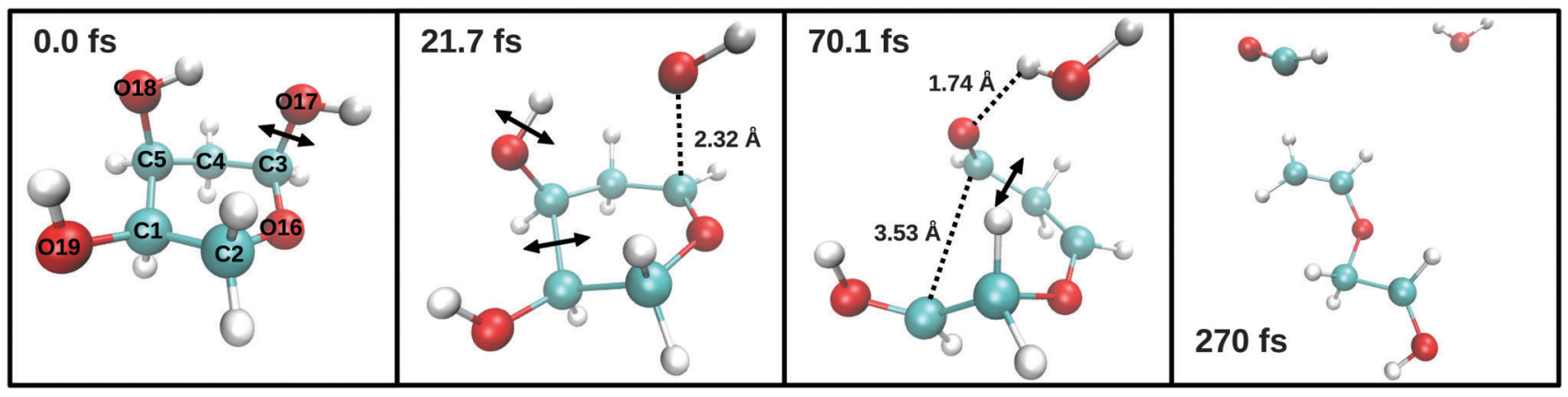

Fig. 5 Fragmentation dynamics of deoxyribopyranose, after a core hole was placed for 9.6 fs on O17. C blue, O red, $\mathrm{H}$ white. 


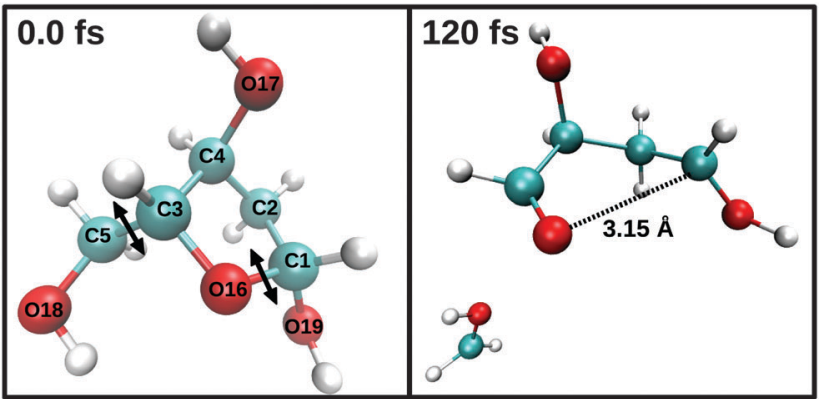

Fig. 6 BOMD trajectory initiated from an electronic configuration where two electrons were removed from the HOMO of the deoxyribofuranose molecule. $\mathrm{C}$ blue, $\mathrm{O}$ red, $\mathrm{H}$ white.

the first $4.8 \mathrm{fs}$, and then return approximately to their initial values at $t=9.6 \mathrm{fs}$.

In contrast, oxygen core-holes $\left(\mathrm{O}^{*}\right)$ induce significant $\mathrm{CO}^{*}$ bond elongation as summarized in Table 2 . The smallest bond elongation is observed in the case of the $\mathrm{O} 16 \mathrm{~K}$-hole, which is located in the furanose ring. This is accompanied by a reduction in the $\mathrm{C} 1 \mathrm{O} 19$ bond by $0.14 \AA$ at $t=9.6 \mathrm{fs}$. The largest increase is observed when a core hole is placed on O19, corresponding to an elongation of the $\mathrm{C} 1 \mathrm{O} 19$ bond by about $0.30 \AA$ at $9.6 \mathrm{fs}$. It also induces variations in the bonds involving $\mathrm{O} 16$.

3.2.3 Double hole dynamics following core ionisation. We studied the core-hole states individually for about $10 \mathrm{fs}$, then switched to the doubly-charged state after either 4.8 or $9.6 \mathrm{fs}$ of the core-hole dynamics. In all cases but one, the fragmentation pattern is the same as that of $\mathrm{dR}^{2+}$ without prior core ionisation. The fragmentation dynamics goes through the following steps: (i) dissociation of $\mathrm{C} 3 \mathrm{C} 5$ bond, which leads to the dissociation of $\mathrm{dR}^{2+}$ into two fragments: $\mathrm{CH}_{3} \mathrm{O}^{+}$with mass $31 \mathrm{amu}$ and $\mathrm{C}_{4} \mathrm{H}_{7} \mathrm{O}_{3}{ }^{+}$ with mass $103 \mathrm{amu}$; and (ii) $\mathrm{C} 1 \mathrm{O} 16$ bond breakage, which leads to the ring opening.

Most interestingly, we observed a new fragmentation pattern when the K-shell vacancy was placed on $\mathrm{O} 18$ during $9.6 \mathrm{fs}$ before the $\mathrm{dR}^{2+}$ dynamics, as shown in Fig. 7 . The trajectory reveals that the $\mathrm{C} 3 \mathrm{C} 4$ and $\mathrm{C} 1 \mathrm{O} 16$ bonds first break at 23.6 and $41.9 \mathrm{fs}$, respectively. Two fragments are thus formed, $\mathrm{C}_{2} \mathrm{H}_{4} \mathrm{O}_{2}$ and $\mathrm{C}_{3} \mathrm{H}_{6} \mathrm{O}_{2}$. The smallest fragment breaks at the $\mathrm{C} 3 \mathrm{C} 5$ bond at $102 \mathrm{fs}$. As a results, this dynamics leads to the formation of $\mathrm{CHO}^{0.25+}$ with mass $29 \mathrm{amu}, \mathrm{CH}_{3} \mathrm{O}^{0.25+}$ with mass $31 \mathrm{amu}$ and $\mathrm{C}_{3} \mathrm{H}_{6} \mathrm{O}_{2}{ }^{1.5+}$ with mass $74 \mathrm{amu}$.

Table 2 Furanose conformation. Oxygen core-hole dynamics

\begin{tabular}{lllll}
\hline \multirow{4}{*}{ Atom } & & \multicolumn{2}{l}{ Bond distance $\AA$} \\
\cline { 3 - 5 } O16 & Bond & $t=0$ & $t=4.8 \mathrm{fs}$ & $t=9.6 \mathrm{fs}$ \\
& C1-O16* & 1.47 & 1.54 & 1.63 \\
& C3-O16* $^{*}$ & 1.49 & 1.51 & 1.65 \\
O17 & C1-O19 & 1.45 & 1.42 & 1.31 \\
O18 & C4-O17* & 1.48 & 1.54 & 1.68 \\
O19 & C5-O18* & 1.44 & 1.50 & 1.64 \\
& C1-O19* & 1.45 & 1.56 & 1.76 \\
& C1-O16 & 1.47 & 1.41 & 1.30 \\
& C3-O16 & 1.49 & 1.52 & 1.57
\end{tabular}

\subsection{Linear conformation}

3.3.1 Double hole dynamics. BOMD of ground state $\mathrm{dR}^{2+}$ were performed for $200 \mathrm{fs}$. The two initial configurations investigated led to different dissociation patterns. The first pathway is illustrated on Fig. 8. The $\mathrm{C} 1 \mathrm{C} 2$ bond dissociates very fast, leading to the formation of a $\mathrm{CH}_{3} \mathrm{O}^{+}$fragment.

In the second pathway, the $\mathrm{C} 1 \mathrm{C} 5$ bond breaks leading to the formation of $\mathrm{C}_{2} \mathrm{O}_{2} \mathrm{H}_{5}{ }^{+}$and $\mathrm{C}_{2} \mathrm{O}_{3} \mathrm{H}_{5}{ }^{+}$fragments.

Here again, the bonds which first break are the CC bonds on which the HOMO presented $\sigma$ character (see Fig. 2), and from which the two electrons were removed.

3.3.2 Double hole dynamics following core ionisation. We have studied the oxygen core-hole states individually for $9.6 \mathrm{fs}$ and then switched to doubly-charged states. We thus generated four trajectories from the same initial configuration as in Fig. 8. Without prior core ionisation, this configuration was shown to lead to the fragmentation of the $\mathrm{dR}^{2+}$ molecule at the $\mathrm{C} 1 \mathrm{C} 2$ bond (see Fig. 8).

The presence of a core vacancy on O17, O18 or O19 during $9.6 \mathrm{fs}$, before the double hole dynamics, led to the same fragmentation pattern as that of $\mathrm{dR}^{2+}$ without the presence of a core hole, that is the one depicted on Fig. 8.

When a core hole is placed on O16 during $9.6 \mathrm{fs}$, the fragmentation pattern was different. As shown on Fig. 9, the C1C5 bond breaks, which is also one of the dissociation patterns found for $\mathrm{dR}^{2+}$ without prior core ionisation, but starting from another initial configuration.

\subsection{Excited states and discussion of results}

Our results can be compared to the experiment by Ha et al., ${ }^{10}$ who irradiated $\mathrm{dR}$ with soft $\mathrm{X}$-rays at energies near the $\mathrm{C}-\mathrm{K}$ and $\mathrm{O}-\mathrm{K}$ edges and measured charged fragments in coincidence. In these gas phase experiments, the most probable conformation is the pyranose one. For this conformation, we found that the main dissociation pathways (see Table 3 ) are either a linearisation of the molecule or the production of $\mathrm{CH}_{2} \mathrm{O}$ and $\mathrm{C}_{4} \mathrm{H}_{8} \mathrm{O}_{3}{ }^{2+}$ fragments. These fragments will not appear on coincidence spectra, as only one of the two fragments is charged. Ha et al. in fact point out that the absence (or weak traces) of fragment $\mathrm{CH}_{2} \mathrm{O}^{+}(30 \mathrm{amu})$ is noteworthy considering that these are most likely the moieties that are the precursors of the $\mathrm{CHO}^{+}$fragments. Here, this fragment is found mostly neutral. It should also be mentioned that $\mathrm{CH}_{x} \mathrm{O}^{+}(x=1, \ldots)$ fragments were shown to desorb from $\mathrm{dR}$ films upon soft X-ray impact. ${ }^{28,29}$ We also find that the $\mathrm{C}_{4} \mathrm{H}_{8} \mathrm{O}_{3}{ }^{2+}$ fragment sometimes dissociates into $\mathrm{C}_{2} \mathrm{H}_{4} \mathrm{O}^{+}(44 \mathrm{amu})$ and $\mathrm{C}_{2} \mathrm{H}_{4} \mathrm{O}_{2}{ }^{+}(60 \mathrm{amu})$. Ha et al. have only observed fragments with mass less than $43 \mathrm{amu}$. It is thus likely that $\mathrm{C}_{2} \mathrm{H}_{4} \mathrm{O}^{+}$and $\mathrm{C}_{2} \mathrm{H}_{4} \mathrm{O}_{2}{ }^{+}$further fragment at longer times, not accessible in terms of the present work trajectory length times. In one case, we show the production of $\mathrm{H}_{2} \mathrm{O}^{0.2+}$ and $\mathrm{CHO}^{0.8+}$. Although the charge of the water molecule is only $+0.2 e$, indicating that it is most often neutral, we find possible (18 amu, $29 \mathrm{amu}$ ) coincidence, as measured by Ha et al. Nonetheless, the most probable pathway is the formation of $\mathrm{CHO}^{+}(29 \mathrm{amu})$ together with $\mathrm{C}_{4} \mathrm{H}_{7} \mathrm{O}_{2}^{+}(87 \mathrm{amu})$. $\mathrm{CHO}^{+}$has been measured in coincidence 


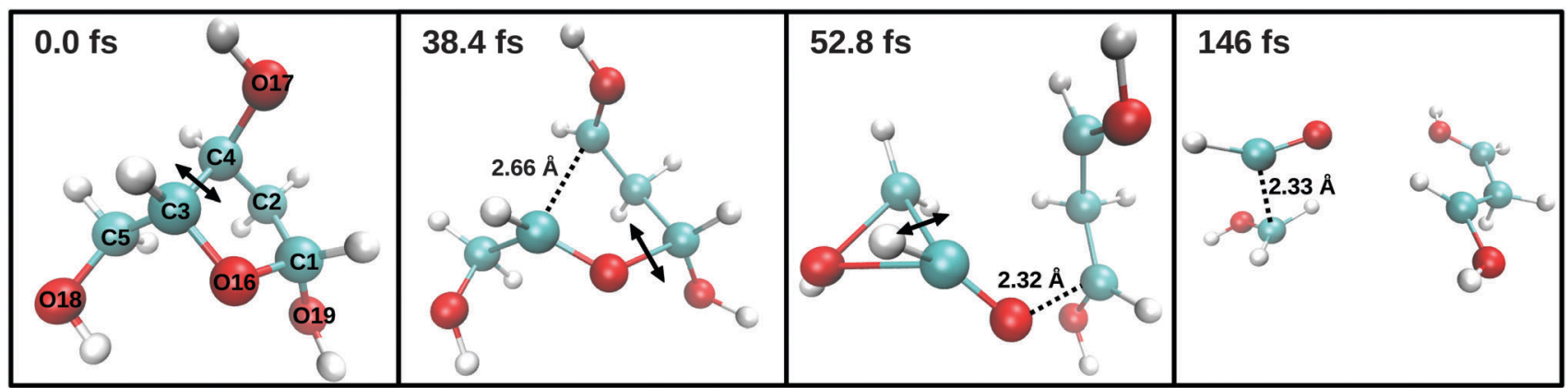

Fig. 7 Fragmentation dynamics of the furanose conformation of $\mathrm{dR}^{2+}$, after a core hole was placed for $9.6 \mathrm{fs}$ on $\mathrm{O} 18$. $\mathrm{C}$ blue, $\mathrm{O}$ red, $\mathrm{H}$ white.

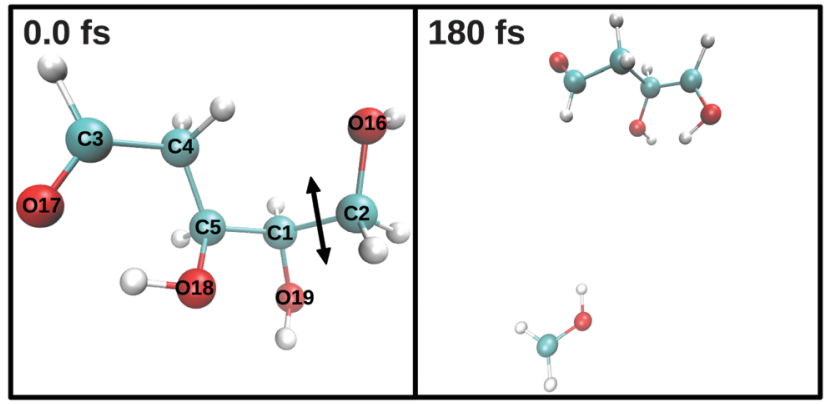

Fig. 8 BOMD trajectory initiated from an electronic configuration where two electrons were removed from the HOMO of the linear $\mathrm{dR}$ conformation. $\mathrm{C}$ blue, $\mathrm{O}$ red, $\mathrm{H}$ white.

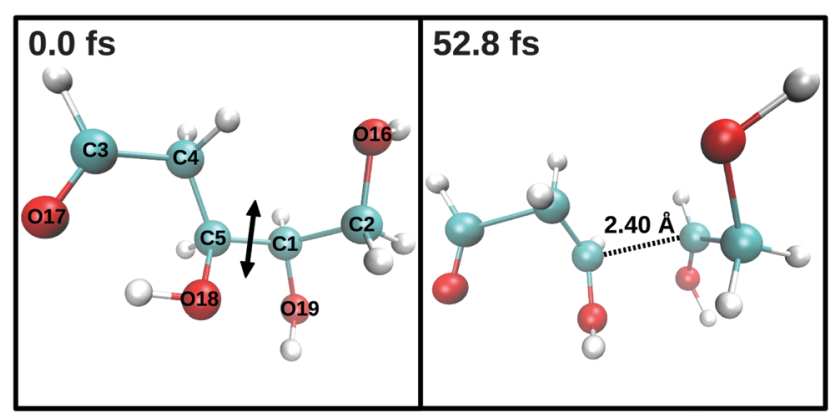

Fig. 9 Fragmentation of the linear conformation of $\mathrm{dR}^{2+}$, after a core hole was placed on $\mathrm{O} 16$ during 9.6 fs. $\mathrm{C}$ blue, $\mathrm{O}$ red, $\mathrm{H}$ white.

with different fragments with masses smaller than 43 amu, which suggests that the 87 amu fragment could undergo fragmentation at longer times.

For the three deoxyribose conformations investigated, the fragmentation induced by the removal of two electrons from the HOMO (without prior core ionisation) appears to be guided by the initial localization of the holes. Fig. 10 shows that the first bond to break is always a CC bond on which the HOMO has a $\sigma$-type: $\mathrm{C} 1 \mathrm{C} 2$ and $\mathrm{C} 1 \mathrm{C} 5$ in the linear case, C1C2 and C1C5 in the pyranose case and C5C3 in the furanose case. In pyranose singly ionised $\mathrm{dR}^{+}$, similar conclusions have been drawn. ${ }^{30}$ Indeed $A b$ Initio MD (AIMD) simulation reveals that most of the fragmentation events start with $\mathrm{C} 1 \mathrm{C} 2$ bond breaking, which is also attributed to the MO shape of removed electron (i.e. the HOMO). Moreover, when the K-hole dynamics before Auger decay is taken into account, we find that the fragmentation pathway after Auger decay is almost always one of the dissociation schemes found for $\mathrm{dR}^{2+}$ without prior core ionisation. Only in two cases, when the core hole was placed on one of the oxygen atom $\left(\mathrm{O}^{*}\right)$ for $9.6 \mathrm{fs}$, was the $\mathrm{CO}^{*}$ bond elongation sufficient to open a new dissociation pathway. Due to the very short lifetime of core vacancies (about 5 fs in the case of oxygen atoms), this will only occur for a small fraction of the core-ionised molecules. As a conclusion, the fragmentation process seems mainly driven by the double-hole state following Auger decay.

To have a complete picture of decay, it would thus be interesting to use a complementary method which allows to follow the dynamics of doubly ionised states in which electrons are ejected from inner valence molecular orbitals. The calculation of the branching ratios of the Auger deexcitation channels would be prohibitive taking into account that the number of 2-combinations from a set of 54 orbitals (in a local spin density approximation picture) is almost 700. Nevertheless, we tested the effect of removing electrons from inner valence molecular orbitals. To that aim, we used Time Dependent-DFT Ehrenferst Molecular Dynamics which allows the description of the nuclear dynamics guided by the evolution of the electronic density, as done in some of our previous works. ${ }^{14-16}$ Since this method is very computationally consuming, we restrict our tests to the furanose conformation and a few location of double holes at the beginning of the dynamics.

The four cases exhibit a common feature (see Fig. 11): the fragmentation is guided by the location of the holes at the beginning of the trajectory, as when the two electrons are removed from the HOMO (see Fig. 10). The fragmentation is more harmful than in the case of double holes in the HOMO, which is not very astonishing in view of the energy deposit in the system (about $20 \mathrm{eV}$ ). This kind of ultrafast multi-fragmentation could explain the fact that no large fragments were measured by Ha et al., ${ }^{10}$ even if the conformation in this experiment is the pyranose one. As a conclusion, the fragmentation appears to be mainly guided by the rearrangement of the electronic density during the deexcitation of the doubly excited ionised state leading to double holes in valence bonds.

This could explain why experimental findings exhibit no difference in fragmentation whether core holes are induced in carbon or oxygen atoms since molecular orbitals are affected in the whole core ionisation and subsequent Auger decay processes. 
Table 3 Different fragmentation patterns

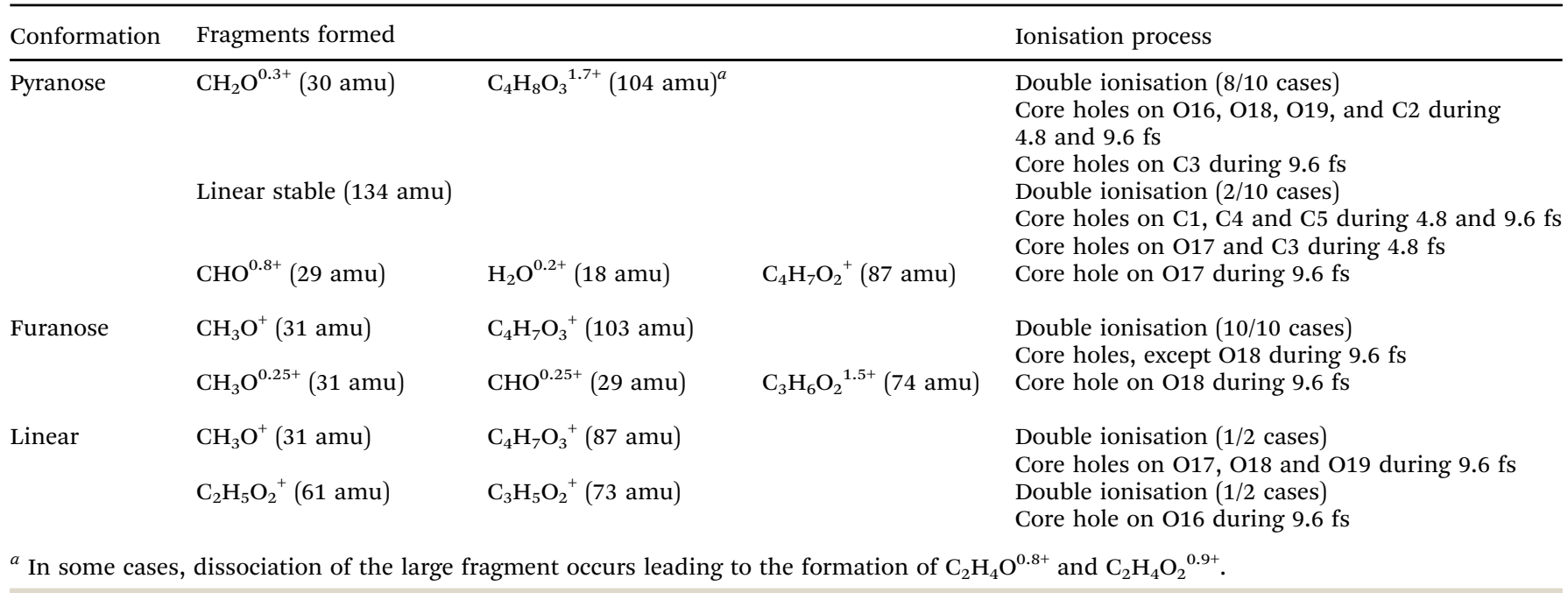

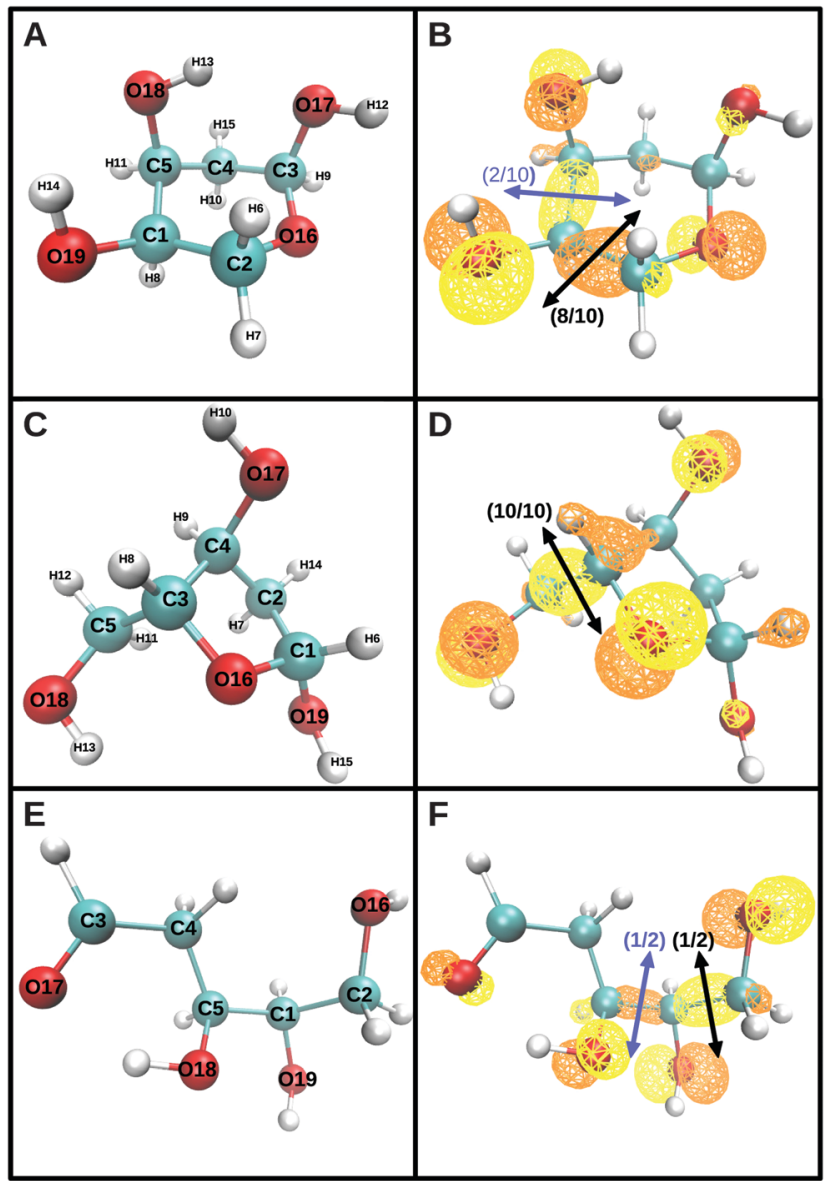

Fig. 10 Fragmentation of the different conformations of $\mathrm{dR}^{2+}$, after double ionisation in the HOMO. The position of the first bond to break is marked by an arrow. In parenthesis, the fraction of the trajectories starting with this bond break divided by the number of initial configurations investigated. $\mathrm{C}$ blue, $\mathrm{O}$ red, $\mathrm{H}$ white.

Several authors ${ }^{10,13}$ have indeed pointed out that the dissociation of a molecule after K-shell ionisation in light atoms and

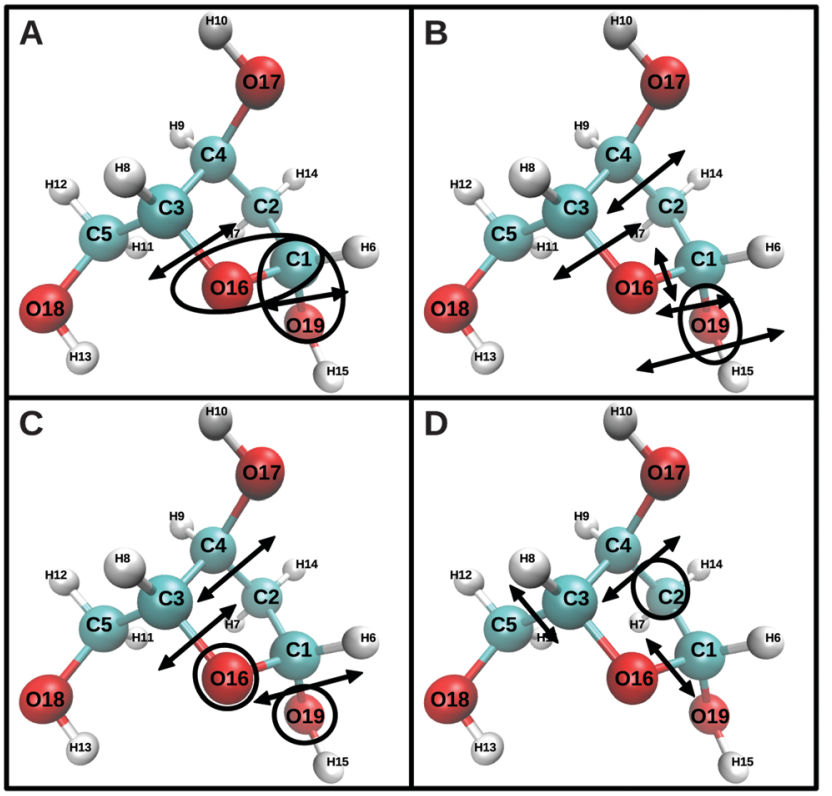

Fig. 11 Fragmentation of furanose $\mathrm{dR}^{2+}$ after removal of deep molecular orbitals with energy (A) $25.9 \mathrm{eV}$, (B) $24.9 \mathrm{eV}$, (C) $24.8 \mathrm{eV}$ and (D) $18.8 \mathrm{eV}$. The localization of the $\mathrm{MO}$ electronic density are schematized as ellipses. Bond breakage positions are marked by arrows. $\mathrm{C}$ blue, $\mathrm{O}$ red, $\mathrm{H}$ white.

Auger decay does not seem to depend on the position nor on the nature of the $\mathrm{K}$ ionised atom. Statistically one cannot directly deduce from the two charged fragments emitted in coincidence where the $\mathrm{K}$ holes were induced by the photoionisation process in this kind of polyatomic molecules. On the contrary, in the case of small symmetrical molecules, where measurements and completely quantum theoretical calculations are possible, dissociation pathways could be deduced as an evidence of preferential bond breaking following Auger decays, with photoelectron-photoionphotoion coincidence technique measurements performed for $\mathrm{C}_{2} \mathrm{H}_{2}$ molecule irradiated at the $\mathrm{C}-\mathrm{K}$ threshold. ${ }^{31}$ In this case, the photoelectron angular distribution (PAD) for non-symmetric 
fragmentation provided direct evidence of a localized corehole and preferential bond breaking following Auger decays. In the case of $\mathrm{dR}$, although core holes are not degenerated in energy (so distinguishable), differences coming from the localization of the $\mathrm{K}$ hole could not be observed in experimental spectra. ${ }^{10}$

The furanose conformation study will provide a reference for future works in which we will (step by step) increase the complexity of the molecule investigated and include a liquid water environment. Future work will consider the effect of adding the base at $\mathrm{C}^{\prime}$ and the phosphate groups at $\mathrm{C} 3^{\prime}$ and $\mathrm{C}^{\prime}$ to the furanose $\mathrm{dR}$ conformation to provide a more realistic model of dissociation of core ionized $\mathrm{dR}$ in the DNA molecule. Also, as pointed out in previous studies on the uracil molecule, the water environment plays a critical role on the fragmentation induced both by core holes ${ }^{26}$ and double ionization events. ${ }^{16}$ We are currently investigating the effect of the liquid water environment on the fragmentation of core-ionized $\mathrm{dR}$.

\section{Conclusions}

For the three conformations investigated, we find the same fragmentation patterns for ground-state doubly-ionised deoxyribose whether or not the core hole dynamics is taken into account before Auger decay. In most cases, no clear memory of the K-hole location neither of the nature of the atoms struck, apart from the energy induced in the molecule, could be found. For the sake of comparison, we have performed TD-DFT Ehrenferst MD which is the only possible method for representing the very excited doubly-ionised states resulting from K-shell ionisation and subsequent Auger effect. Such a method cannot be used for statistics because of the too large computational cost necessary. But these preliminary dynamics reinforce the conclusion that the main ingredient for the dissociation is the excited state, i.e. the localization of the holes in the molecule, after Auger decay. We have indeed previously shown that doubly-ionised states, created by removing electrons in MO with very near energy but localized in different bonds, lead to very different bond breakage in the case of isolated uracil. ${ }^{15}$ This fact shows that the energy deposited in the molecule is not the unique criteria to take into account for the fate of a doubly ionised molecule. Moreover, in the gas phase, such polyatomic molecules do not exhibit very large bond elongations during the core hole dynamics, before Auger decay. Here, specific fragmentation was only found in two cases, when the core vacancy was located on an oxygen atom external to the cycle for $9.6 \mathrm{fs}$. Due to the very short lifetime of $\mathrm{O}-\mathrm{K}$ vacancies (about $5 \mathrm{fs}$ ), this will only occur for a small fraction of the core-ionised molecules. As a conclusion, the location of the $\mathrm{K}$ hole does not play a major role in elongating bonds within the cycle in such cyclic polyatomic molecules. The location of the core hole will play a major role to induce Auger effect thus 2 holes in different orbitals of the molecule. The fragmentation will not only depend on the energy but also strongly on the location of the empty orbitals thus created.

\section{Acknowledgements}

We thank Y. Jeanvoine and J. Y. Salpin for helpful discussions on sugar conformations. This work was performed using HPC resources from GENCI-CINES/IDRIS (Grant 2014-085014) and CCRE UPMC. All calculations have been performed with the CPMD package, Copyright IBM Corp 1990-2011, Copyright MPI fuer Festkoer-perforschung Stuttgart 1997-2001. The present study is partially supported by the Reimei Research Program (Japan Atomic Energy Agency).

\section{References}

1 S. Saigusa, Y. Ejima, K. Kobayashi and M. S. Sasaki, Int. J. Radiat. Biol., 1992, 61, 785-790.

2 M. Watanabe, M. Suzuki, K. Watanabe, K. Suzuki, N. Usami, A. Yokoya and K. Kobayashi, Int. J. Radiat. Biol., 1992, 61, 161-168.

3 M.-A. Hervé du Penhoat, B. Fayard, F. Abel, A. Touati, F. Gobert, I. Despiney-Bailly, M. Ricoul, L. Sabatier, D. L. Stevens, M. A. Hill, D. T. Goodhead and A. Chetioui, Radiat. Res., 1999, 151, 649-658.

4 B. Fayard, A. Touati, F. Abel, M.-A. Hervé du Penhoat, I. DespineyBailly, F. Gobert, M. Ricoul, A. L'Hoir, M.-F. Politis, M. A. Hill, D. L. Stevens, L. Sabatier, E. Sage, D. T. Goodhead and A. Chetioui, Radiat. Res., 2002, 157, 128-140.

5 F.-N. Gobert, M. Lamoureux, M.-A. Hervé du Penhoat, M. Ricoul, A. Boissière, A. Touati, F. Abel, M.-F. Politis, B. Fayard, J. Guigner, L. Martins, I. Testard, L. Sabatier and A. Chetioui, Int. J. Radiat. Biol., 2004, 80, 135-145.

6 M.-A. Hervé du Penhoat, A. Eschenbrenner, F. Abel, A. Boissière, J.-M. Guigner, A. Chetioui, M.-F. Politis, A. Touati, E. Sage, T. J. Jenner, D. L. Stevens and M. A. Hill, Int. J. Radiat. Biol., 2010, 86, 205-219.

7 J. Campbell and T. PAPP, At. Data Nucl. Data Tables, 2001, 77, 1-56.

8 F. Alvarado, S. Bari, R. Hoekstra and T. Schlatholter, Phys. Chem. Chem. Phys., 2006, 8, 1922-1928.

9 F. Alvarado, J. Bernard, B. Li, R. Brédy, L. Chen, R. Hoekstra, S. Martin and T. Schlathölter, ChemPhysChem, 2008, 9, 1254-1258.

10 D. Ha, M. Huels, M. Huttula, S. Urpelainen and E. Kukk, Phys. Rev. A: At., Mol., Opt. Phys., 2011, 84, 033419.

11 T. Sekitani, E. Ikenaga, K. Tanaka, K. Mase, M. Nagasono, S. Tanaka and T. Urisu, Surf. Sci., 1997, 390, 107-111.

12 E. Sako, Y. Kanameda, E. Ikenaga, M. Mitani, O. Takahashi, K. Saito, S. Iwata, S. Wada, T. Sekitani and K. Tanaka, J. Electron Spectrosc. Relat. Phenom., 2001, 114-116, 591-596.

13 P. Bolognesi, M. Castrovilli, P. O’Keeffe, A. Casavola, D. Catone, S. Turchini and L. Avaldi, Nucl. Instrum. Methods Phys. Res., Sect. B, 2012, 279, 118-123.

14 I. Tavernelli, M.-P. Gaigeot, R. Vuilleumier, C. Stia, M.-A. Hervé du Penhoat and M.-F. Politis, ChemPhysChem, 2008, 9, 2099-2103.

15 P. López-Tarifa, M.-A. Hervé du Penhoat, R. Vuilleumier, M.-P. Gaigeot, I. Tavernelli, A. L. Padellec, J.-P. Champeaux, 
M. Alcamí, P. Moretto-Capelle, F. Martín and M.-F. Politis, Phys. Rev. Lett., 2011, 107, 023202.

16 P. López-Tarifa, M.-P. Gaigeot, R. Vuilleumier, I. Tavernelli, M. Alcamí, F. Martín, M.-A. Hervé du Penhoat and M.-F. Politis, Angew. Chem., Int. Ed., 2013, 52, 3160-3163.

17 J.-P. Champeaux, P. Çarçabal, M. Sence, P. Moretto-Capelle and P. Cafarelli, J. Phys. B: At., Mol. Opt. Phys., 2011, 44, 045205.

18 E. Kukk, D. T. Ha, Y. Wang, D. G. Piekarski, S. Diaz-Tendero, K. Kooser, E. Itälä, H. Levola, M. Alcamí, E. Rachlew and F. Martín, Phys. Rev. A: At., Mol., Opt. Phys., 2015, 91, 043417. 19 M.-A. Hervé du Penhoat, P. López-Tarifa, K. K. Ghose, Y. Jeanvoine, M.-P. Gaigeot, R. Vuilleumier, M.-F. Politis and M.-C. Bacchus-Montabonel, J. Mol. Model., 2014, 20, 2221.

20 L. Guler, Y.-Q. Yu and H. Kenttämaa, J. Phys. Chem. A, 2002, 106, 6754-6764.

21 R. Car and M. Parrinello, Phys. Rev. Lett., 1985, 55, 2471-2474. CPMD, http://www.cpmd.org/, Copyright IBM Corp 1990-2015, Copyright MPI für Festkörperforschung Stuttgart 1997-2001.

22 N. Troullier and J. Martins, Phys. Rev. B: Condens. Matter Mater. Phys., 1991, 43, 1993-2006.
23 A. D. Becke, Phys. Rev. A: At., Mol., Opt. Phys., 1988, 38, 3098-3100.

24 C. Lee, W. Yang and R. Parr, Phys. Rev. B: Condens. Matter Mater. Phys., 1988, 37, 785-789.

25 R. F. W. Bader, Atoms in Molecules - A Quantum Theory, Oxford University Press, Oxford, UK, 1990.

26 C. R. Stia, M.-P. Gaigeot, R. Vuilleumier, A. O. Fojón, M.-A. Hervé du Penhoat and M.-F. Politis, Eur. Phys. J. D, 2010, 60, 77-83.

27 O. Takahashi, M. Odelius, D. Nordlund, A. Nilsson, H. Bluhm and L. G. M. Pettersson, J. Chem. Phys., 2006, 124, 064307.

28 K. Fujii, K. Akamatsu and A. Yokoya, Surf. Sci., 2003, 528, 249-254.

29 K. Fujii, K. Akamatsu and A. Yokoya, Radiat. Res., 2004, 161, 435-441.

30 D. Ghosh, A. Golan, L. Takahashi, A. Krylov and M. Ahmed, J. Phys. Chem. Lett., 2012, 3, 97-101.

31 J. Adachi, K. Hosaka, T. Teramoto, M. Yamazaki, N. Watanabe, M. Takahashi and A. Yagishita, J. Phys. B: At., Mol. Opt. Phys., 2007, 40, F285. 